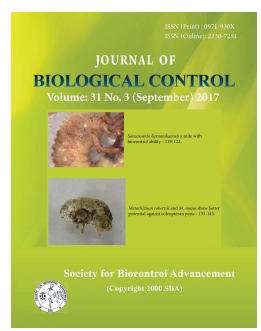

\title{
Metarhizium majus and Metarhizium robertsii show enhanced activity against the coleopteran pests Holotricha serrata and Oryctes rhinoceros
}

\author{
V. VELAVAN ${ }^{*}$, G. SIVAKUMAR ${ }^{1}$ R. RANGESHWARAN ${ }^{1}$, R. SUNDARARAJ ${ }^{2}$ and T. O. SASIDHARAN ${ }^{3}$ \\ ${ }^{1}$ ICAR-National Bureau of Agricultural Insects Resources, Bangalore - 560024, Karnataka, India \\ ${ }^{2}$ Institute of Wood Science and Technology, Bangalore - 560 003, Karnataka \\ ${ }^{3}$ Ashoka Trust for Research in Ecology and the Environment (ATREE), Bangalore - 560064, Karnataka, India \\ *Corresponding authorE-mail: velavan.sv@gmail.com
}

\begin{abstract}
Studies were conducted to systematically isolate Metarhizium isolates from the insect cadavers and soils of South India. Morphological and PCR amplified sequences of 5.8S ITS regions and RNA polymerase II largest subunit (RPB1) gene regions were used to identify the isolates at species level. Eight Metarhizium isolates were isolated and initially identified by morphological and microscopic studies. Further identification was confirmed through 5.8SrRNA ITS and RPB1 analysis. They were identified as three isolates of $M$. robertsii J.F. Bisch., Rehner \& Humber sp. nov. (ArMz3R, ArMz3S and ArMz6W), one isolate of M. majus (J.R. Johnst.) J.F. Bisch., Rehner \& Humber (VjMz1W) and four isolates of M. anisopliae (WnMz1S, NlMz2S, BgMz2S and DhMz4R). Topical conidial suspensions (TCS) and powder based formulations (PBF) of the eight indigenous isolates of Metarhizium spp. that were isolated from insect cadavers and soils of South India were tested against coleopteran pests Holotricha serrata L. and Oryctes rhinoceros L. that cause serious damage to sugarcane and palm trees respectively. Against $H$. serrata TCS of $M$. robertsii (ArMz6W) was the most effective with an $\mathrm{LC}_{50}$ of $6.893 \times 10^{5} \mathrm{cfu} / \mathrm{ml}$ and caused $100 \%$ mortality against the $3^{\text {rd }}$ instar larvae in 5 days; PBF elicited an $\mathrm{LC}_{50}$ of $7.502 \times 10^{5} \mathrm{cfu} / \mathrm{ml}$ with $96 \%$ mortality in 10 days. Against O. rhinoceros TCS $\left(\mathrm{LC}_{50}\right.$ of $\left.9.75 \times 10^{5} \mathrm{cfu} / \mathrm{ml}\right)$ of $M$. majus $(\mathrm{VjMz} 1 \mathrm{~W})$ caused $90 \%$ mortality in 7 days and the $\mathrm{PBF}\left(\mathrm{LC}_{50}\right.$ of $9.57 \times 10^{5}$ $\mathrm{cfu} / \mathrm{ml}$ ) caused $86 \%$ mortality in 14 days. The results establish that $M$. robertsii is highly effective against $H$. serrata and against $O$. rhinoceros M. majus was the most effective. The TCS formulations of these two strains can be readily deployed for field applications.
\end{abstract}

KEY WORDS: Holotricha serrata, Metarhizium spp. Oryctes rhinoceros, Powder based formulation (PBF), Topical conidial suspension (TCS)

(Article chronicle: Received: 24.08.2017; Revised: 28.09.2017; Accepted: 30.09.2017)

\section{INTRODUCTION}

Holtrichia serrata (Fabricius) commonly known as white grub is a serious pest of many agricultural crops of South India, it causes serious damage to sugarcane roots. They survive by feeding and mating in sugarcane root system and damage is caused by the larvae (Anitha et al., 2006). Apart from sugarcane they are also serious pests of groundnut and potato. $H$. serrata are widely disturbed around the southern states viz. Tamil Nadu, Karnataka, Kerala, Andhra Pradesh (Veeresh, 1977). Another serious pest is the rhinoceros beetle Oryctes rhinoceros L. (Scarabaeidae: Dynastinae) and can be devastating to coconut not only in India but across Southeast Asia leading to severe economic losses (Nair et al., 1997; Norman and Basri, 1997; Bedford, 2014;). As with many beetles, adults and larvae have different feeding preferences. Young adults of $O$. rhinoceros feed on healthy leaves and larvae feed on rotting plant material in soil.

The entomopathogenic fungus, M. anisopliae, discovered 100 years ago by Mednichoff has a rather wide host range and is widely used as a microbial 
biocontrol agent on various types of pests, which include insects from different orders: Lepidoptera, Coleoptera, Hemiptera, Diptera, Orthoptera, Hymenoptera, as well as non-insect arthropods like elm bark beetle, mosquito larvae, plant hoppers, coconut leaf beetle, rhinoceros beetle, onion thrips, storage pests, white grub and cattle tick. There are 60 commercial mycoinsecticides based on $M$. anisopliae strains (Shanmugam et al., 2014; Chandal et al., 2015). There are several studies conducted to prove the efficacy of Metarhizium against many insect pests, they are mostly based on M. anisopliae. Against Holotrichia serrata strains of $M$. anisopliae have been shown to cause infection and formulations have been used in sugarcane fields to bring down the white grub population (Srikanth et al., 2011; Shanmugam et al., 2014; Sathayaraj and Karthick 2008; Thamarai Chelvi et al., 2011; Pradya and Mohit, 2014). M. anisopliae have been tested for control scarab species of several coleopteran beetles (Hurpin and Robert, 1972). O. rhinoceros is a serious pest of oil palm in Malaysia (Bedford, 2014; Norman and Basri, 1997), and formulations of $M$. anisopliae were used for biological control (Ramle et al., 2009).

Since most of the research work is based on $M$. anisopliae strains, reports on the effectiveness of other Metarhizium species are very limited. In the present study systematic survey was done to collect forest soil and insect cadaver samples for isolation of Metarhizium species and to see if apart from $M$. anisopliae whether other species could be more effective in control of insect pests such as $O$. rhinoceros and $H$. serrata.

\section{MATERIALS AND METHODS}

\section{Survey and collection of the Metarhizium isolates}

The survey was made to various forests of South India (Tamilnadu, Kerala, Karnataka and Andhra Pradesh) dead insects and soil samples were collected from these forests (wet evergreen, moist deciduous, dry deciduous and scrub forest) (Table-1). Metarhizium was isolated from insect cadaver and from soil using Galleria larvae as bait. Infected cadavers and Galleria larvae were surface-sterilized by dipping sequentially in $70 \%$ ethyl alcohol, $1 \%$ sodium hypochlorite, and sterile distilled water, each for 2-3 minutes. They were dissected and placed on potato dextrose agar plus yeast extract (PDAY medium containing (1\% yeast extract $0.6 \mathrm{~g}, 100 \mu \mathrm{g} / \mathrm{ml}$ Chloramphenicol, $50 \mu \mathrm{g} / \mathrm{ml}$ Streptomycin, 2 mg crystal violet) and incubated at 28$1^{\circ} \mathrm{C}$ and $90 \% \mathrm{RH}$ to facilitate growth and sporulation of the fungus. The purification of the Metarhizium spp. was done using Veen's medium (Veen and Ferron, 1966; Hu and St. Leger, 2002). Final purification was done by hyphal tip method. Plates were incubated for 7 to 14 days at $27^{\circ} \mathrm{C}$ to induce growth and sporulation of the fungus. After 15 days emerged mycelia were harvested by scraping off the content from each Petri plate. Slants of Metarhizium fungal cultures were prepared and stored. Matarhizium spp. were initially described based on morphological, conidiophores, and phialides mounted microscopes. Further identification was done by sequence analysis of $5.8 \mathrm{~S}$ rRNA of the internal transcribed spacer (ITS) and RNA polymerase II largest subunit (RPB1) as per protocols by Bischoff et al. (2009) and Nishi et al. (2011) (Table 1).

\section{Production of Metarhizium spp.}

Topical conidial suspension (TCS) was prepared as per the procedure described by Fleming (1968) and Tashiro et al. (1973). Powder based formulation (PBF) was prepared as per the procedure of Samiyappen et al. (2003) and Ramle et al. (2009). TCS was prepared by scraping out the mycelia from 10-15 days old culture grown PDA in Petri plates in an aqueous solution of $0.02 \%$ Tween 80 and $1 \%$ glycerol, with continuous stirring in a tube and filtered through a single layer of linen to remove debris and mycelia. The conidial concentration was estimated with haemocytometer under light microscope. Subsequently the spore suspension was diluted to make a final suspension of $1 \times 10^{5}$ spores $/ \mathrm{ml}$ with $0.02 \%$ Tween 80 . PBF was prepared by harvesting 15 days old mycelia grown in Potato dextrose broth (PDB). Harvesting was done centrifugation at $10,000 \mathrm{rpm}$ for $15 \mathrm{~min}$. The pellet was mixed @100g in mixture containing 200g rice flour, Yeast extract 50g, 10ml glycerol, $10 \mathrm{ml}$ honey and 
$630 \mathrm{~g}$ of talc.

\section{Collection and rearing of white grubs and Oryctes rhinoceros}

Adults and larvae of Holotrichia serrata and Oryctes rhinoceros were collected from agricultural fields (palm, rice and banana and sugarcane) in NorthThanjavur district, Sathanur Village Latitude and Longitude: $10.82035 \mathrm{~N}$ : $79.08925 \mathrm{E}$ and surrounding areas in plastic boxes $(23 \times 20 \mathrm{~cm}$ diameter $\mathrm{x}$ height $)$ containing the native soil. In the laboratory larvae, pupae and adults were separated into individual plastic boxes and kept at $27^{\circ} \mathrm{C}$ and $70 \% \mathrm{RH}$. The feed consisted of organic matter, decaying wood trash carrot, potato tree yam, ground nuts and other debris. The rearing room was protected from direct sun-light and UV-light by making it dark. The boxes were covered with muslin cloth and tied with rubber bands to ensure good aeration. The moisture level was maintained through wet cloth and soil with gentle moistening when the soil felt dry. While rearing of the grubs, the following biological parameters such as, date of collection, date and causes of grub mortality, date of pupation and adult emergence were recorded.
After six week of rearing, healthy grubs of $3^{\text {rd }}$ instar larvae were used in bioassay.

\section{Bioassay against Holotrichia serrata, Oryctes rhinoceros larvae}

Third instar larvae of Holotrichia serrata and Oryctes rhinoceros were transferred aseptically to fresh plastic boxes ( $4 \mathrm{~cm}$ diameter and $6 \mathrm{~cm}$ height). Each box contained moist soil medium with some humus and potato tubers as food. Already prepared TCS/PBF were tested at three concentrations $\left(1 \times 10^{5}, 1 \times 10^{4}\right.$ and $1 \times 10^{3}$ spores $/ \mathrm{ml}$ ). Into each box $5 \mathrm{ml}$ suspension was added by droplets onto the larvae. Three replications were maintained and total larvae tested for each treatment was fifteen. For control sterile water with $0.02 \%$ sterile water was used. Observations on mortality was recorded up to 7 days.

\section{Statistical analysis}

Cumulative mortality at the end of the experiment was analysed and $\mathrm{LC}_{50}$ were determined using the probit analysis program. SAS 15.0 for windows.
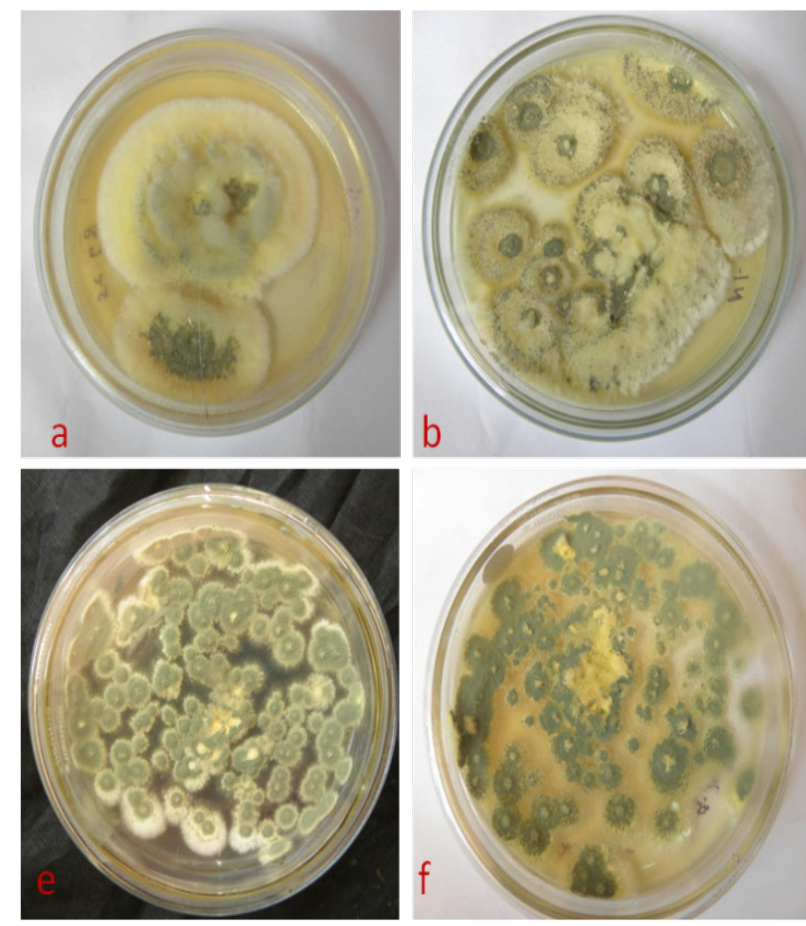
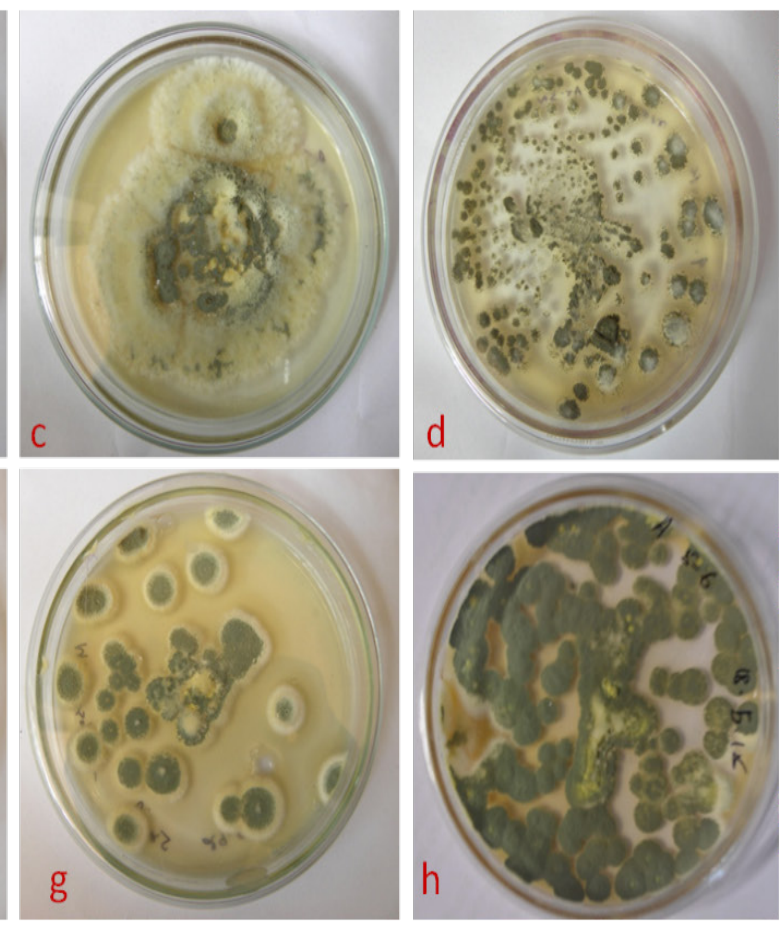

Fig. 1. Metarhizium colonies grown on PDAY at room temperature for 15 days (a) M. robertsii (ArMz3R), (b) M. robertsii (ArMz3R), (c). M. robertsii (ArMz6W), (d). M. majus (e). M. anisopliae (f). M. anisopliae, (g). M. anisopliae and (h). M. anisopliae. 

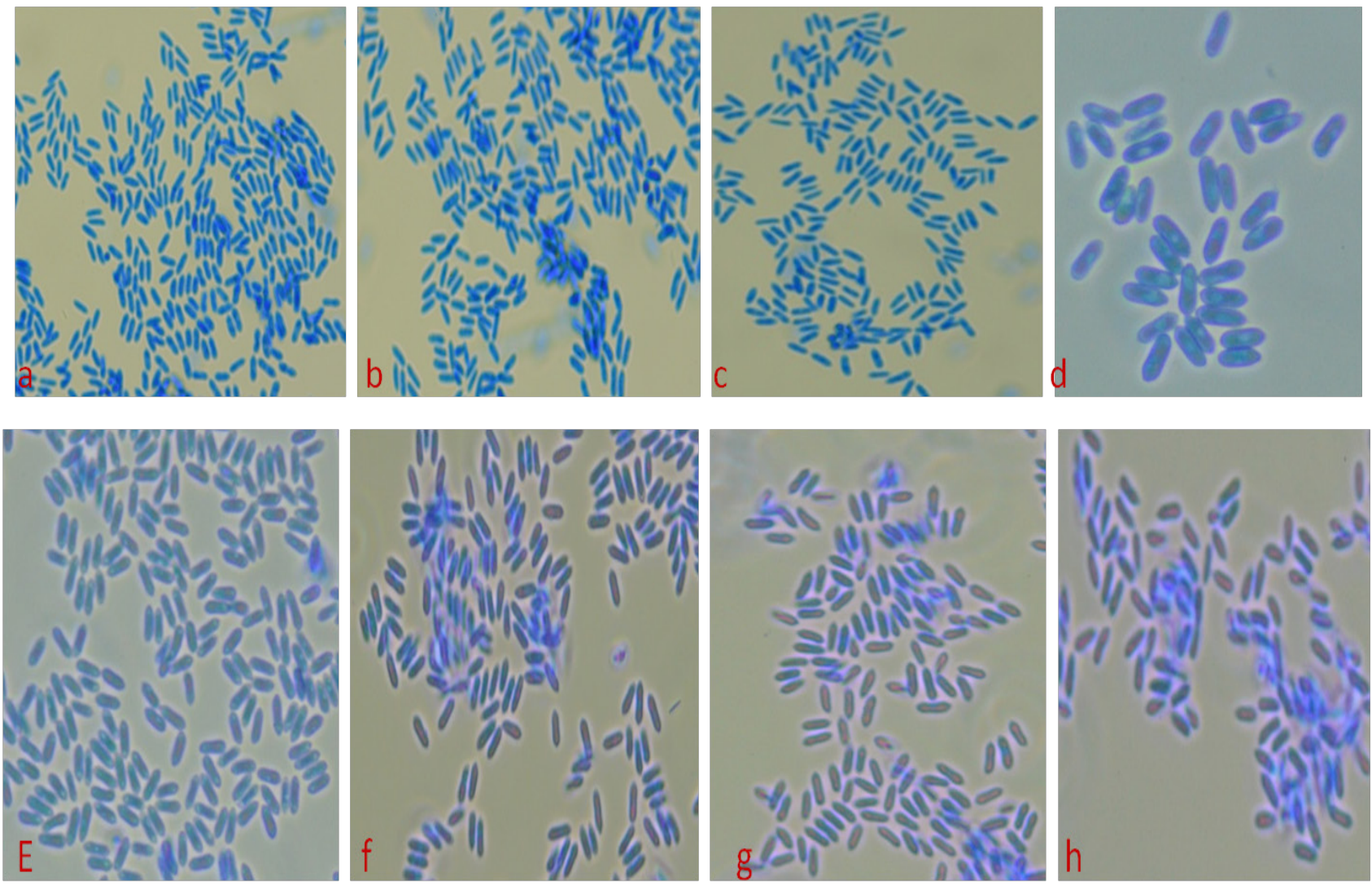

Fig. 2. The morphology of conidia under bright field light microscope (100X); (a-c), Metarhizium robertsii conidia small, (d), M. majus conidia large, (e-h), M. anisopliae conidia medium.

\section{RESULTS AND DISCUSSION}

Experiments were conducted to collect forest soil and insect cadaver samples for isolation of Metarhizium species and to see if apart from M. anisopliae whether other species could be more effective in control of insect pests such as $O$. rhinoceros and $H$. serrata. Eight Metarhizium isolates were isolated and initially identified by morphological and microscopic studies (Table 1 and Fig. 1-2) as per conidial shape and size (Driver et al., 2000; Bischoff et al., 2009). Further identification was confirmed through 5.8SrRNA ITS and RPB1 analysis. They were identified as three isolates of $M$. robertsii (ArMz3R, ArMz3S and ArMz6W), one isolate of M. majus (VjMzlW) and four isolates of $M$. anisopliae (WnMz1S, NlMz2S, BgMz2S and DhMz4R). The partial sequences for 5.8SrRNA ITS and RPB1 were submitted to NCBI (Table 1). Bischoff et al. (2009) employed a multigene phylogenetic approach from nuclear encoded EF-1, RPB1, RPB2 and - tubulin gene regions and proposed nine terminal taxa in the $M$. anisopliae complex which included M. majus and M. robertsii. Similarly Nishi et al. (2011) analyzed the internal transcribed spacer (ITS) and 50 end of the EF-1a sequence of 145 isolates of Metarhizium spp. isolated from soil and e identified six species: M. anisopliae, M. brunneum, M. guizhouense, M. majus, M. pingshaense and $M$. robertisii. In our studies morphological, 5.8SrRNA ITS and RPB1 analysis was done to confirm the species.

\section{Bioassay against Oryctes rhinoceros}

Bioassay was conducted against $3^{\text {rd }}$ instar larvae of Oryctes rhinoceros with TCS formulation containing the eight Metarhizium isolates. It was observed that at 7 days after treatment the highest mortality (90\%) and infection (86\%) was with Metarhizium majus treated 


\begin{tabular}{|c|c|c|c|c|c|c|c|c|c|c|}
\hline 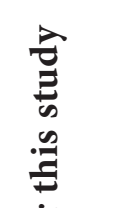 & 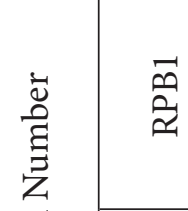 & $\begin{array}{l}\hat{m} \\
\text { ô } \\
\infty \\
0 \\
2 \\
2\end{array}$ & 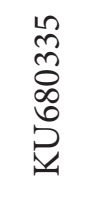 & 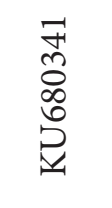 & 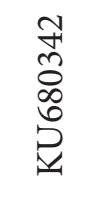 & 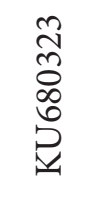 & 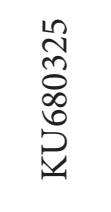 & 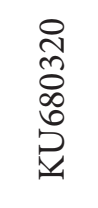 & 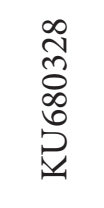 & \multirow{2}{*}{ 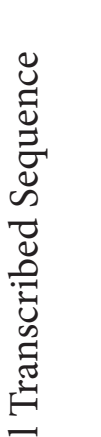 } \\
\hline 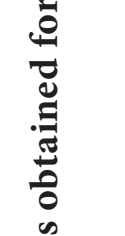 & 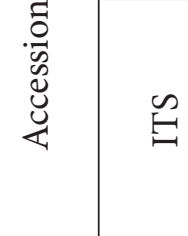 & 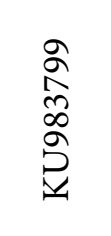 & 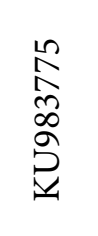 & 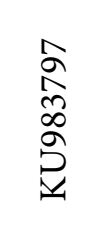 & 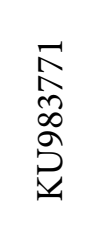 & $\begin{array}{l}\infty \\
\infty \\
\infty \\
\infty \\
\infty \\
2 \\
2\end{array}$ & $\begin{array}{l}n \\
\infty \\
\infty \\
\infty \\
\infty \\
\stackrel{2}{2}\end{array}$ & $\begin{array}{l}8 \\
\infty \\
\hat{N} \\
\infty \\
2 \\
2 \\
2\end{array}$ & $\begin{array}{l}+ \\
\infty \\
\infty \\
\infty \\
\infty \\
2 \\
2\end{array}$ & \\
\hline 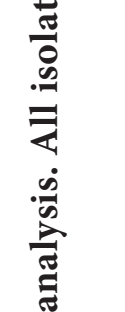 & 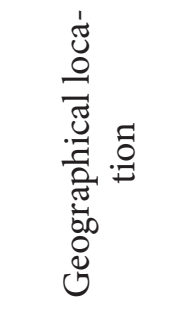 & 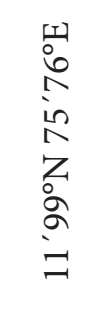 & 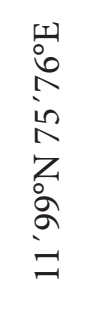 & $\begin{array}{l}\text { 니 } \\
\text { o } \\
\text { in } \\
\text { in } \\
\text { z } \\
\text { à } \\
= \\
=\end{array}$ & 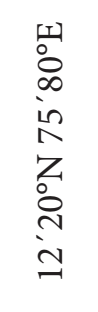 & $\begin{array}{l}11 \\
0 \\
0 \\
0 \\
i \\
0 \\
7 \\
0 \\
0 \\
= \\
=\end{array}$ & 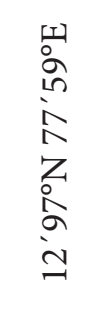 & 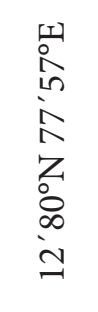 & 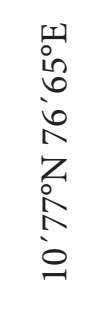 & 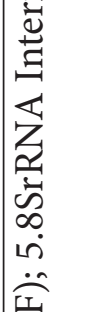 \\
\hline 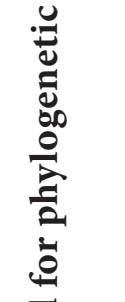 & 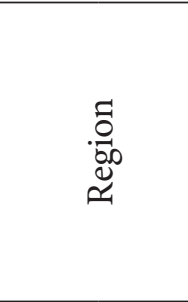 & 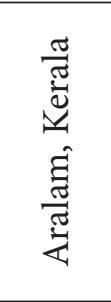 & 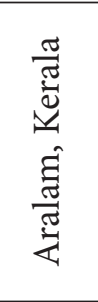 & 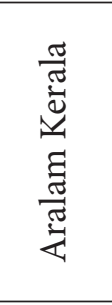 & 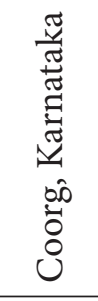 & 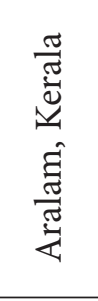 & 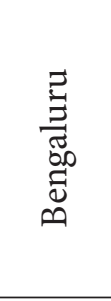 & 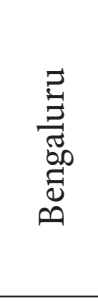 & 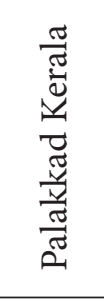 & 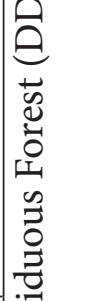 \\
\hline 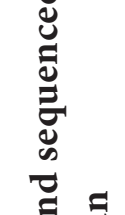 & 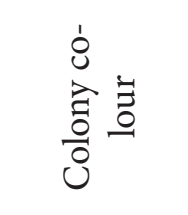 & 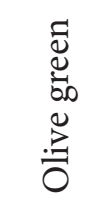 & 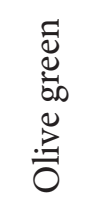 & 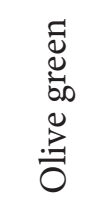 & 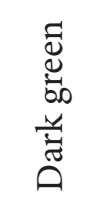 & 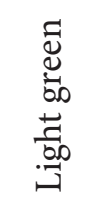 & 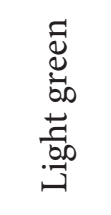 & 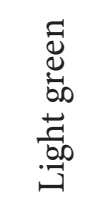 & 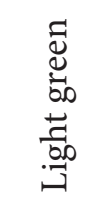 & 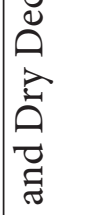 \\
\hline 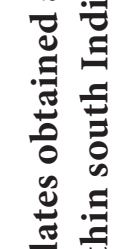 & 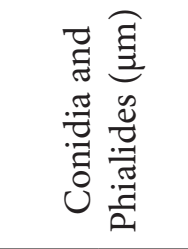 & 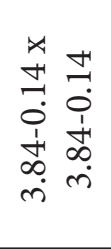 & 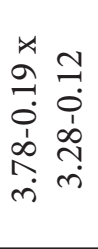 & 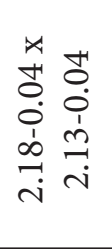 & 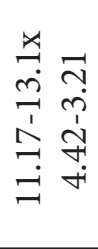 & 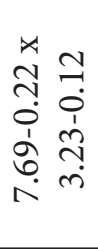 & 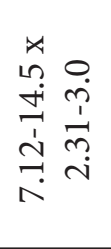 & 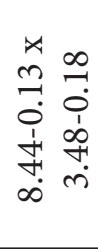 & 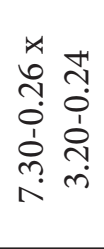 & 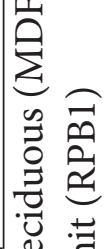 \\
\hline $\begin{array}{l}\stackrel{0}{3} \\
\stackrel{0}{3} \\
\stackrel{0}{2} \\
\frac{0}{2}\end{array}$ & 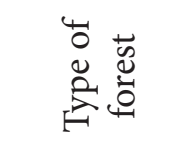 & $\stackrel{4}{3}$ & $\stackrel{4}{3}$ & $\stackrel{5}{3}$ & $\frac{1}{3}$ & $\stackrel{4}{3}$ & $\stackrel{\mathrm{I}}{\mathrm{i}}$ & 岎 & $\stackrel{\mathrm{L}}{\mathrm{i}}$ & 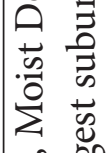 \\
\hline 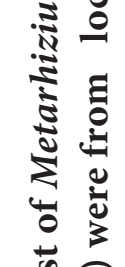 & 点 & 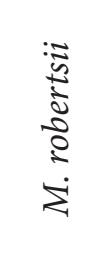 & 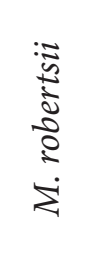 & 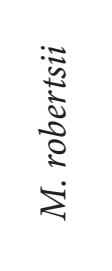 & 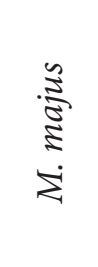 & 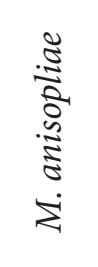 & 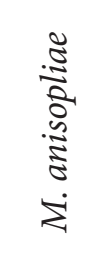 & 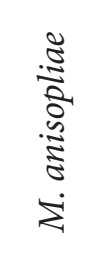 & 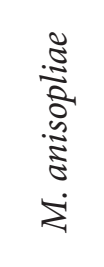 & 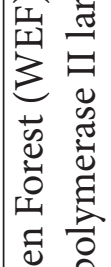 \\
\hline$\stackrel{0}{-\overrightarrow{0}}$ & 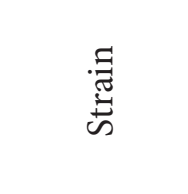 & $\sum_{i=1}^{\sqrt[n]{\pi}}$ & $\sum_{i=1}^{\infty}$ & $\sum_{\substack{i \\
4}}^{3}$ & $\sum_{i=1}^{3}$ & $\sum_{\substack{n \\
3}}^{\infty}$ & $\sum_{\bar{Z}}^{\stackrel{N}{Z}}$ & $\sum_{\substack{\infty \\
N}}^{\mathbb{N}}$ & 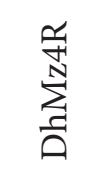 & 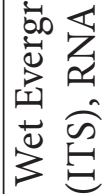 \\
\hline
\end{tabular}


Table 2. Probit analysis of mortality response in field-collected third instar larvae of Oryctes rhinoceros following treatment with TCS of Metarhizium

\begin{tabular}{|c|c|c|c|c|c|c|c|c|}
\hline Metarhizium & Mortality & Infection & $\mathrm{LC}_{50}$ & \multicolumn{2}{|c|}{ 95\% Fiducial limits } & Slop \pm SE & $\mathrm{X} 2$ & $\mathrm{P}$ \\
\hline $\begin{array}{l}\text { M. robertsii } \\
\text { (ArMz3R) }\end{array}$ & 84 & 56 & 14.78 & 10.8037 & 21.5689 & $1.106 \pm 0.634$ & 6.42 & 0.0113 \\
\hline $\begin{array}{l}\text { M. robertsii } \\
\text { (ArMz3S) }\end{array}$ & 80 & 46 & 14.86 & 6.9628 & 74.6436 & $1.092 \pm 0.069$ & 4.09 & 0.0430 \\
\hline $\begin{array}{l}\text { M. robertsii } \\
\text { (ArMz6W) }\end{array}$ & 76 & 53 & 17.69 & 14.4800 & 31.9684 & $1.143 \pm 0.857$ & 7.34 & 0.0067 \\
\hline $\begin{array}{l}\text { M. majus } \\
\text { (VjMz1W) }\end{array}$ & 90 & 86 & 9.75 & 4.2387 & 12.0897 & $1.168 \pm 1.047$ & 8.16 & 0.0043 \\
\hline $\begin{array}{l}\text { M. anisopliae } \\
\text { (WnMz1S) }\end{array}$ & 66 & 43 & 21.10 & 16.9789 & 56.0096 & $1.195 \pm 0.876$ & 7.25 & 0.0071 \\
\hline $\begin{array}{l}\text { M. anisopliae } \\
\text { (NlMz2S) }\end{array}$ & 66 & 40 & 25.68 & 19.3167 & 24.9661 & $1.291 \pm 0.592$ & 5.85 & 0.0156 \\
\hline $\begin{array}{l}\text { M. anisopliae } \\
\text { (BgMz1S ) }\end{array}$ & 67 & 43 & 18.62 & 15.4102 & 33.2553 & $1.162 \pm 1.059$ & 8.24 & 0.0041 \\
\hline $\begin{array}{c}\text { M. anisopliae } \\
\text { (DhMz4R) }\end{array}$ & 70 & 43 & 18.55 & 16.1216 & 24.7683 & $1.298 \pm 2.178$ & 13.24 & 0.0003 \\
\hline
\end{tabular}

${ }^{\star} \mathrm{LC}_{50}$ values are expressed as $\times 10^{5}$ spores $/ \mathrm{ml}$; TCS $=$ topical conidial suspension

and the $\mathrm{LC}_{50}$ was calculated as $9.75 \times 10^{5}$ conidia/ ml. Significant mortality was also observed with $M$. robertsii (ArMz3R) treated wherein $84 \%$ mortality was seen with a $\mathrm{LC}_{50}$ value of $14.78 \times 10^{5}$ condia $/ \mathrm{ml}$. Surprisingly $M$. anisopliae isolates were less virulent (Table 2 and Fig. 3). When tested with PBF again $M$. majus (VjMz1W) was the most virulent with highest mortality of $90 \%$ in 10 days and the $\mathrm{LC}_{50}$ was $9.57 \times 10^{5}$ spores $/ \mathrm{ml}$. similar mortality (90\%) was observed with $M$. robertsii (ArMz3S) treated and the $\mathrm{LC}_{50}$ was $11.98 \times 10^{5}$ spores $/ \mathrm{ml}$. Two $M$. anisopliae isolates (WnMz1S and DhMz4R) exhibited 86\% mortality with $\mathrm{LC}_{50}$ ranging from 14.18 to $19.12 \times 10^{5}$ spores/ $\mathrm{ml}$ (Table 3 and Fig. 3). The results establish that $M$. majus (VjMz1W) was an ideal candidate to combat O. rhinoceros. This is the first report to indicate the usefulness of $M$. majus and $M$. robertsii in biological control. Reports are available in using $M$. anisopliae against $O$. rhinoceros, $M$. anisopliae formulated in powder form caused $90 \%$ mortality and $73 \%$ infection and the fungus could infect all stages of $O$. rhinoceros larvae (Ramle et al., 2007). Powder formulations of M. anisopliae based on kaolin and rice were tested under laboratory conditions and 93\% mortality was 
Table 3. Probit analysis of mortality response in field-collected third instar larvae of Oryctes rhinoceros following treatment with PBF of Metarhizium

\begin{tabular}{|c|c|c|c|c|c|c|c|c|}
\hline $\begin{array}{c}\text { Metarhizium } \\
\text { species }\end{array}$ & $\begin{array}{c}\text { Mortality } \\
\%\end{array}$ & $\begin{array}{c}\text { Infection } \\
\%\end{array}$ & $\mathrm{LC}_{50}$ & \multicolumn{2}{|c|}{$\begin{array}{c}95 \% \text { Fiducial } \\
\text { Limits }\end{array}$} & Slop \pm SE & $\mathrm{X}^{2}$ & $\mathrm{P}$ \\
\hline $\begin{array}{c}\text { M. robertsii } \\
\text { (ArMz3R) }\end{array}$ & 83 & 53 & 17.78 & 14.0107 & 57.2927 & $1.118 \pm 0.414$ & 5.43 & 0.0198 \\
\hline $\begin{array}{c}\text { M. robertsii } \\
\text { (ArMz3S) }\end{array}$ & 90 & 60 & 11.98 & 3.9459 & 15.3456 & $1.104 \pm 0.396$ & 5.38 & 0.0204 \\
\hline $\begin{array}{c}\text { M. robertsii } \\
\text { (ArMz6W) }\end{array}$ & 86 & 56 & 13.62 & 11.5536 & 15.5961 & $1.180 \pm 2.620$ & 17.47 & 0.0001 \\
\hline $\begin{array}{c}\text { M. majus } \\
\text { (VjMz1W) }\end{array}$ & 90 & 70 & 9.57 & 0.6618 & 12.5190 & $1.126 \pm 0.327$ & 5.07 & 0.0244 \\
\hline $\begin{array}{c}\text { M. anisopliae } \\
\text { (WnMz1S) }\end{array}$ & 86 & 52 & 14.18 & 11.2460 & 17.4497 & $1.128 \pm 1.376$ & 10.11 & 0.0015 \\
\hline $\begin{array}{c}\text { M. anisopliae } \\
\text { (NlMz2S) }\end{array}$ & 85 & 55 & 16.47 & 12.5825 & 40.5832 & $1.107 \pm 0.384$ & 5.32 & 0.0210 \\
\hline $\begin{array}{c}\text { M. anisopliae } \\
\text { (BgMz1S) }\end{array}$ & 80 & 50 & 19.97 & 16.9391 & 31.0107 & $1.280 \pm 1.745$ & 11.04 & 0.0009 \\
\hline $\begin{array}{c}\text { M. anisopliae } \\
\text { (DhMz4R) }\end{array}$ & 86 & 46 & 19.12 & 16.1844 & 29.4487 & $1.224 \pm 1.571$ & 10.52 & 0.0012 \\
\hline
\end{tabular}

${ }^{\star} \mathrm{LC}_{50}$ values are expressed as $\times 10^{5}$ spores $/ \mathrm{ml} ; \mathrm{PBF}=$ powder based formulation
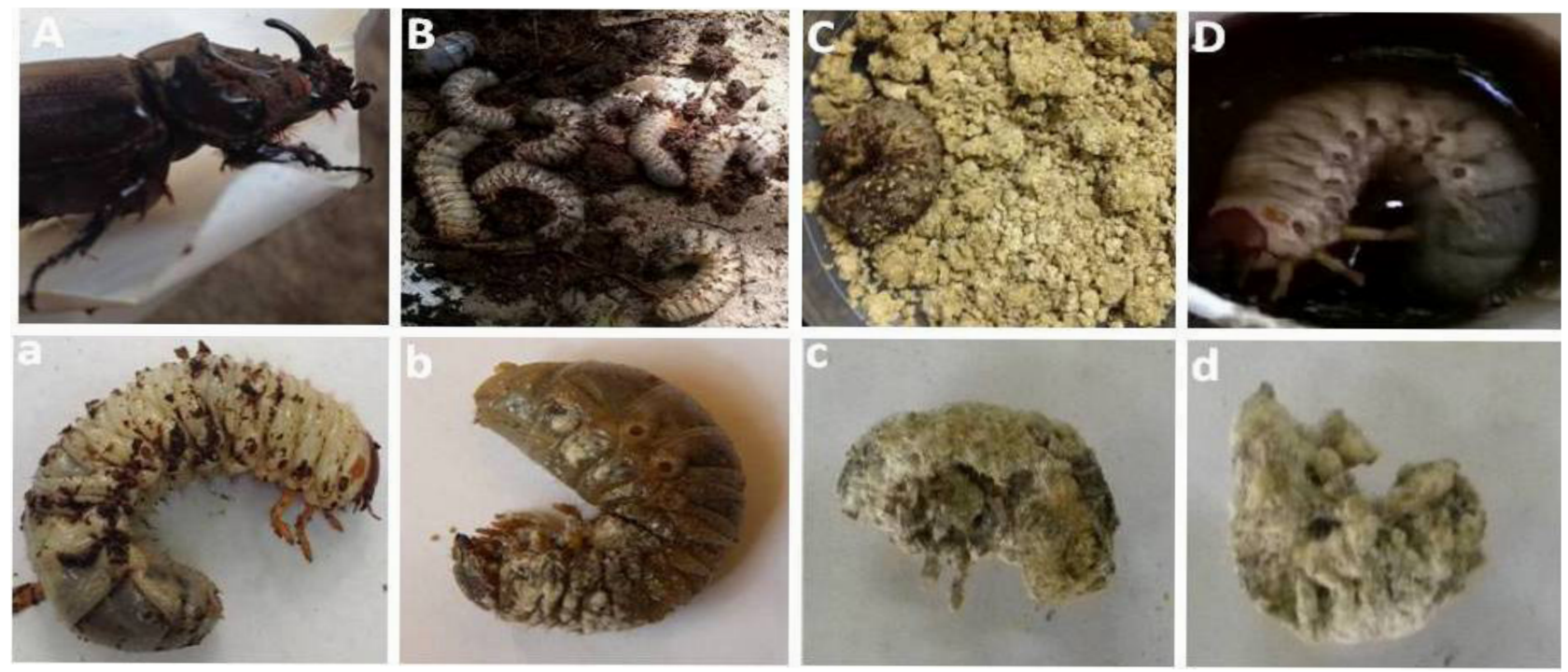

Fig. 3. Bioassy against Oryctes rhinoceros: (A). O. rhinoceros adult beetle, (B). Breeding site in decaying vermin composting, (C). Healthy third-instar larvae, (D), Treatment with Conidial suspesnion, (a). 3rd and 4th instar larvae, (b-c). Dead infected larvae, (d). Larvae mummified with mycelia and conidia of Metarhizium majus. 
observed in 18 days (Ramle et al., 2009).Liquid based conidial suspension @ 300ml/L of water was effective in reducing O. rhinoceros (Verma, 2013)

\section{Bioassay against Holotrichia serrata}

The eight Metarhizium isolates were also evaluated against $3^{\text {rd }}$ instar larvae of Holotrichia serrata with TCS and EBF formulations. With TCS it was observed that at 5 days after treatment the highest mortality (100\%) and infection (95-90\%) was observed with all the three Metarhizium robertsii treated and the $\mathrm{LC}_{50}$ was calculated as 6.89 to $9.75 \times 10^{5}$ conidia/ml (Table 4 and Fig. 4). Significant mortality (94-96\%) was also observed with M. anisopliae (WnMz1S and DhMz4R) and M. majus (VjMz1W) treated larvae of $H$. serrata. However the $M$. robertsii strains displayed better virulence and strain ArMz6W was the most toxic. The bioassay with PBF formulation also gave similar results and 93-96\% mortality was observed with all the three $M$. robertsii strains and the $\mathrm{LC}_{50}$ ranged as 7.50 to $9.50 \times 10^{5}$ spores/ml. High mortality (90\%) was also observed with remaining Metarhizium isolates with $\mathrm{LC}_{50}$ ranging from 17.10 to $23.60 \times 10^{5}$ spores $/ \mathrm{ml}$ (Table 5 and Fig. 4). The results establish the fact that M. robertsii strains will be better choice for combating H. serrata.

Table 4. Probit analysis of mortality response in field-collected third instar larvae of Holotrichia serrata following treatment with TCS of different Metarhizium spp

\begin{tabular}{|c|c|c|c|c|c|c|c|c|}
\hline $\begin{array}{c}\text { Metarhizium } \\
\text { species }\end{array}$ & $\begin{array}{c}\text { Mortality } \\
\%\end{array}$ & $\begin{array}{c}\text { Infection } \\
\%\end{array}$ & $\mathrm{LC}_{50}$ & \multicolumn{2}{|c|}{$\begin{array}{c}95 \% \text { Fiducial } \\
\text { Limits }\end{array}$} & Slop \pm SE & X2 & P \\
\hline $\begin{array}{c}\text { M. robertsii } \\
\text { (ArMz3R) }\end{array}$ & 100 & 90 & 9.753 & 4.2387 & 12.089 & $1.1687 \pm 1.0473$ & 8.16 & 0.0043 \\
\hline $\begin{array}{c}\text { M. robertsii } \\
\text { (ArMz3S) }\end{array}$ & 100 & 93 & 8.172 & 4.3859 & 9.9131 & $1.6899 \pm 2.2494$ & 10.83 & 0.0010 \\
\hline $\begin{array}{c}\text { M. robertsii } \\
\text { (ArMz6W) }\end{array}$ & 100 & 95 & 6.893 & 0.4011 & 9.6811 & $1.3499 \pm 0.6167$ & 5.84 & 0.0157 \\
\hline $\begin{array}{c}\text { M. majus } \\
\text { (VjMz1W) }\end{array}$ & 95 & 85 & 11.63 & 9.90417 & 12.974 & $1.3290 \pm 3.8535$ & 23.61 & 0.0001 \\
\hline $\begin{array}{c}\text { M. anisopliae } \\
\text { (WnMz1S) }\end{array}$ & 92 & 74 & 14.20 & 11.7666 & 16.897 & $1.1482 \pm 1.8791$ & 12.93 & 0.0003 \\
\hline $\begin{array}{c}\text { M. anisopliae } \\
\text { (NlMz2S) }\end{array}$ & 94 & 76 & 13.82 & 10.1142 & 17.523 & $1.1142 \pm 0.9702$ & 8.01 & 0.0046 \\
\hline $\begin{array}{c}\text { M. anisopliae } \\
\text { (BgMz1S })\end{array}$ & 96 & 78 & 11.10 & 2.87657 & 14.063 & $1.1135 \pm 0.4321$ & 5.51 & 0.0189 \\
\hline $\begin{array}{c}\text { M. anisopliae } \\
\text { (DhMz4R) }\end{array}$ & 94 & 76 & 12.84 & 9.4172 & 15.365 & $1.1274 \pm 1.3667$ & 10.06 & 0.0015 \\
\hline
\end{tabular}

${ }^{\star} \mathrm{LC}_{50}$ values are expressed as $\times 10^{5}$ spores $/ \mathrm{ml}$; TCS $=$ topical conidial suspension 
Table 5. Probit analysis of mortality response in field-collected third instar larvae of Holotrichia serrata following treatment with PBF of different Metarhizium spp.

\begin{tabular}{|c|c|c|c|c|c|c|c|c|}
\hline & $\begin{array}{c}\text { Mortality } \\
\%\end{array}$ & $\begin{array}{c}\text { Infection } \\
\%\end{array}$ & $\mathrm{LC}_{50}$ & \multicolumn{2}{c|}{$\begin{array}{c}95 \% \text { Fiducial } \\
\text { Limits }\end{array}$} & Slop \pm SE & $\mathrm{X} 2$ & $\mathrm{P}$ \\
\hline $\begin{array}{c}\text { M.robertsii } \\
\text { (ArMz3R) }\end{array}$ & 93 & 73 & 9.478 & 6.2739 & 11.1821 & $1.3471 \pm 2.3362$ & 13.65 & 0.0002 \\
\hline $\begin{array}{c}\text { M.robertsii } \\
\text { (ArMz3S) }\end{array}$ & 95 & 75 & 8.134 & 3.0253 & 10.3511 & $1.3433 \pm 1.3380$ & 8.74 & 0.0031 \\
\hline $\begin{array}{c}\text { M.robertsii } \\
\text { (ArMz6W) }\end{array}$ & 96 & 76 & 7.502 & 1.3249 & 10.0135 & $1.3696 \pm 0.8926$ & 6.82 & 0.0090 \\
\hline $\begin{array}{c}\text { M.majus } \\
\text { (VjMz1W) }\end{array}$ & 90 & 73 & 17.118 & 13.893 & 41.7293 & $1.5405 \pm 3.1498$ & 6.80 & 0.0091 \\
\hline $\begin{array}{c}\text { M.anisopliae } \\
\text { (WnMz1S) }\end{array}$ & 90 & 70 & 17.102 & 14.139 & 31.4180 & $1.5096 \pm 3.5736$ & 8.75 & 0.0030 \\
\hline $\begin{array}{c}\text { M.anisopliae } \\
\text { (NlMz2S) }\end{array}$ & 90 & 70 & 17.148 & 14.640 & 24.9644 & $1.5089 \pm 2.8179$ & 10.20 & 0.0010 \\
\hline $\begin{array}{c}\text { M.anisopliae } \\
\text { (BgMz1S) }\end{array}$ & 90 & 63 & 18.375 & 16.093 & 23.7808 & $1.5022 \pm 3.8275$ & 21.00 & 0.0001 \\
\hline $\begin{array}{c}\text { M.anisopliae } \\
\text { (DhMz4R) }\end{array}$ & 90 & 53 & 23.610 & 18.061 & 80.2086 & $1.3402 \pm 1.0473$ & 8.16 & 0.0040 \\
\hline
\end{tabular}

${ }^{\star} \mathrm{LC}_{50}$ values are expressed as $\times 10^{5}$ spores $/ \mathrm{ml} ; \mathrm{PBF}=$ powder based formulation

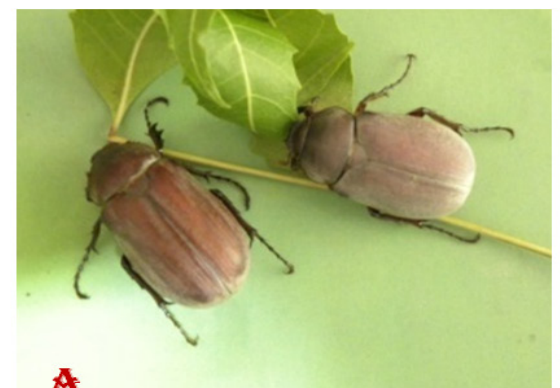

A

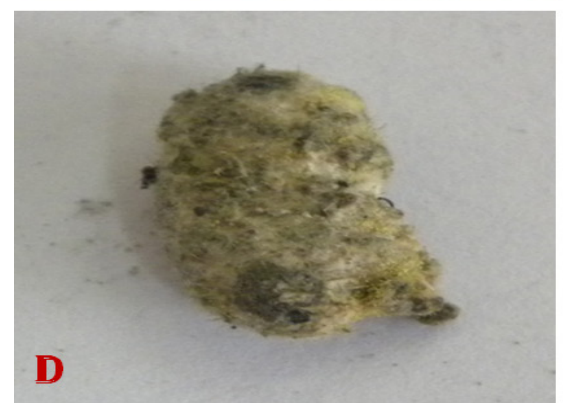

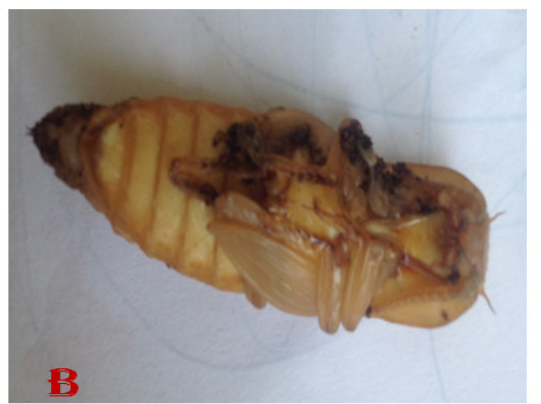

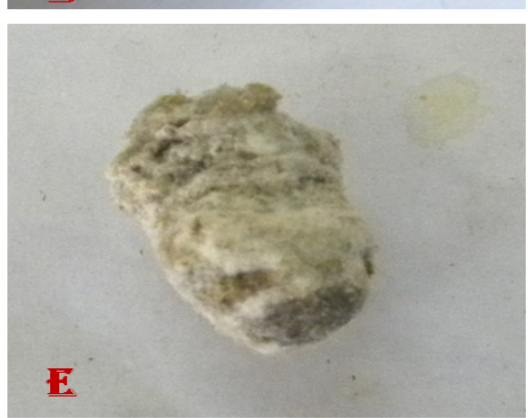

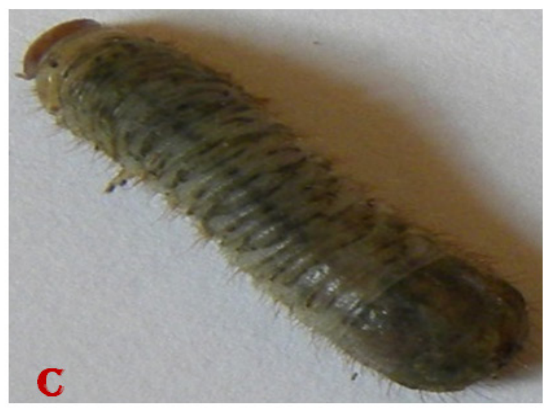

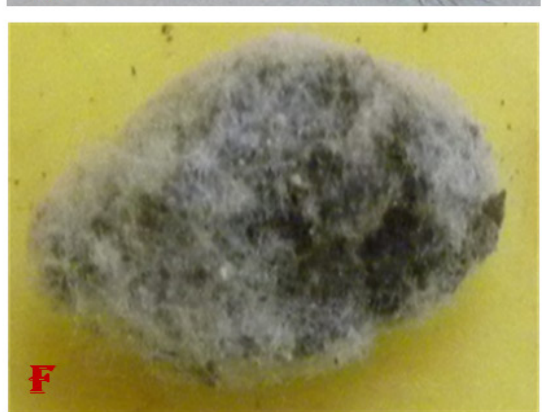

Fig. 4. Bioassay against Holotrichia serrata (A-B). Adult H. serrata (C). Healthy uninfected larvae in control, (D-F). Insect cadavers mummified with mycelia and conidia of Metarhizium robertsii. 
Reports are available in using M. anisopliae against Holotrichia serrata. Thamari Chelvi et al. (2011) M. anisopliae against $H$. serrata and obtained a mean mortality of $81 \%$ with liquid formulation containing $1 \times 10^{8}$ conidia/ml and $76-78 \%$ mortality was obtained with powder formulations containing $4 \times 10^{8}$ spores/g in 15 days. Srikanth et al. (2011) also used conidial suspension @ and obtained $\mathrm{LC}_{50}$ of $9.28 \times 10^{7}$ conidia/ $\mathrm{ml}$ in 7 days. Powder formulations of $M$. anisopliae species were used against lepidopteran or coleopteran larvae primarily employed the method of surface contamination with conidial suspension followed by provisioning of food such as roots or shoots in containers (Easwaramoorthy et al. 2004; Srikanth et al. 2006; Manisegaran et al., 2011) and application of M. anisopliae @ $4 \times 10^{9}$ conida $/ \mathrm{ml}$ gaveg $92 \%$ control of white grub population at 30 days. Talc based on powder formulation of M. anisopliae @ $1 \times 10^{5}$ spores/ $\mathrm{ml}$ gave highest virulence and mortality against $H$. serrata (Thamari Chelvi et al., 2011).

The present study indicate that $M$. majus (VjMz1W) was highly virulent against $O$. rhinoceros and all the isolates of $M$. robertsii proved to be more effective against $H$. serrata. They performed better than the $M$. anisopliae strains as seen in the $\mathrm{LC}_{50}$ values. There is no literature available on the use of M. majus or M. robertsii in biological control. This is the first study to show that these could be exploited along with $M$. anisopliae for the management of $O$. rhinoceros and $H$. serrata. Further studies like lytic enzyme profiling and toxin nature of $M$. majus and $M$. robertsii will throw a better light on their virulence.

\section{ACKNOWLEDGEMENT}

The authors are grateful to the Department of Biotechnology, New Delhi for providing financial support to carry out this work during 2013-2016. We also thank the Director, ATREE, IWST and Director, ICAR-NBAIR, Bangalore for providing facilities to undertake the study. The permission granted by the Principal Chief Conservator of Forests of Karnataka, Kerala, Tamil Nadu and Andhra Pradesh to undertake the survey is duly acknowledged.

\section{REFERENCES}

Anitha V, Rogersb DJ, Wightmanc J, Wardd A. 2006. Distribution and abundance of white grubs (Coleoptera: Scarabaeidae) on groundnut in southern India. Crop Prot. 25: 732-740. Crossref.

Bedford GO. 2013. Biology and Management of Palm dynastid Beetles: Recent Advances. Ann Rev Entomol. 58: 353-372. Crossref. PMid:23317044

Bischoff SA, Rehner SA, Humber RA. 2009. A Multilocus pylogeny of Metarhizium anisopliae lineage. Mycologia 101: 512-530. Crossref. PMid:19623931

Chandel RS, Mandeep Pathania1 KS, Verma BB, Sumit V, Vinod Kumar. 2015. The Ecology and Control of Potato, Whitegrubs of India. Potato Res. 58: 147-164. Crossref.

Driver F, Milner RJ, Trueman WH. 2000. A taxonomic revision of Metarhizium based on a phylogenetic analysis of rDNA sequence data. Mycol Res. 104 (2): 134-150. Crossref.

Easwaramoorthy S, Srikanth J, Santhalakshmi G, Geetha N. 2004. Laboratory and field studies on Beauveria brongniartii (Sacc.) Petch, against Holotrichia serrata F. (Coleoptera: Scarabaeidae) in sugarcane. Proceedings of the Annual Convention Sugar Technologists Association of India 66: 3-A19.

Fleming WE. 1968. Biological control of the Japanese beetle. USDA-ARS Tech Bull No. 1383. 78.

Hurpin B, Robert PH. 1972. Comparison of the activity of certain pathogens of the cockchafer Melolontha melolontha in plots of natural meadowland. J Invertebr Pathol. 19: 291-298. Crossref.

Hu G, st. Leger RJ. 2002. Field studies using a recombinant mycoinsecticide (Metarhizium anisopliae) reveal that it is rhizosphere competent. Appl Environ Microbiol. 68: 6383-6387. Crossref. PMid:12450863 PMCid:PMC134390

Metschnikoff VE. 1884. Ueber eine Sprosspilzkrankheit der Daphnien. Beitrag zur Lehre über den 
Kampf der Phagocyten gegen Krankheit serreger. Virc Arcv. 96: 177-195.

Nair CPR, Danielm M, Ponnamma KN. 1997. Integrated Pest Management in palms; Nambiar KKN, Nair MK (Eds.). Coconut Development Board, Kochi, India 30 pp.

Nishi O, Iiyama K, Yasunaga-Aoki C, Shimizu S. 2011. Incongruence between EF-1a Phylogeny and Morphology of Metarhizium majus and Metarhizium guizhouense in Japan. Entomotech. 34: 19-23.

Pradnya BM, Pandurang BM. 2014. Efficacy of newer molecules of insecticides against white grub in sugarcane. Asian J Bio Sci. 9:173-177. Crossref.

Ramle M, Norman K, Ang, BN, Ramlah Ali SA, Basri MW. 2007. Application of powder formulation of Metarhizium anisopliae to control Oryctes rhinoceros in rotting oil palm residues under leguminous cover crops. J Oil Palm Res. 19: 318-330.

Ramle M, Norman K, Mohd BW. 2009. Pathogenicity of granule formulations of Metarhizium anisopliae against the larvae of the oil palm rhinoceros beetle, Oryctes rhinoceros (L.). J Oil Palm Res. 2: 602-612.

Sahayaraj K, Karthick S. 2008. Mass production of entomopathogenic fungi using agricultural products and by products. Afr J Biotechnol. 7(12): 1907-1910. Crossref.

Shanmugam PS, Saravanan NA, Tamilselvan N. 2014. Assessment of biofungicides, Beauveria brongniartii and Metarhizium anisopliae against sugarcane white grub, Holotrichia serrata, Ind Jou Plant Prot. 42(4): 467-469.
Srikanth J, Santhalakshmi G, Tamizharasi V. 2006. Viability and virulence of selected Beauveria brongniartii formulations against Holotrichia serrata. Sugar Tech. 8(2\&3): 152-154. Crossref.

Srikanth J, Santhalakshmi G, Nirmala R. 2011. An improved bioassay method for entomopathogenic fungi of sugarcane pests and its evaluation in studies of virulence in subcultures. Sugar Tech. 13(2): 156-165. Crossref.

Tashiro H. 1973. Bionomics and control of root feeding insect pests: grubs and billbugs. Bull Entomol Soc Am. 9: 92-94. Crossref.

Thamarai Chelvi C, Thilagaraj RW, Nalini R. 2011. Field efficacy of formulations of microbial insecticide Metarhizium anisopliae (Hyphocreales: Clavicipitaceae) for the control of sugarcane white grub Holotrichia serrata F (Coleoptera :Scarabidae). J Biopest. 4(2): 186-189.4

Veen KH, Ferron P. 1966. A selective medium for isolation of Beauveria tenella and of Metarrhizium anisopliae. J Invertebr Pathol. 8: 268-269. Crossref.

Veeresh GK, Veerarajan, PV, Gubbaiah R, Vijaymohan N. 1977. Efficacy of certain soil insecticides against root grubs ( $H$. serrata F.) under irrigated and unirrigated conditions in tropical soils. Proceedings All India Symposiums on Soil Biology and Ecology, (2), pp. 23-31, 1977.

Yamini Varma CK. 2013. Efficacy of ecofriendly management against Rhinoceros beetle grubs in coconut. J Biopest. 6(2): 101-103 